\title{
Corrigendum
}

\section{Corrigendum to "Global Stability of Malaria Transmission Dynamics Model with Logistic Growth"}

\author{
Abadi Abay Gebremeskel (iD \\ Department of Mathematics, Haramaya University, Haramaya, Ethiopia \\ Correspondence should be addressed to Abadi Abay Gebremeskel; abaybeti@yahoo.com \\ Received 16 September 2018; Accepted 19 September 2018; Published 18 October 2018 \\ Copyright (c) 2018 Abadi Abay Gebremeskel. This is an open access article distributed under the Creative Commons Attribution \\ License, which permits unrestricted use, distribution, and reproduction in any medium, provided the original work is properly \\ cited.
}

In the article titled "Global Stability of Malaria Transmission Dynamics Model with Logistic Growth" [1], there was a sign error on the global stability analysis of the endemic equilibrium point where a preceding sign should be minus $(-)$ instead of plus (+) in equation (34) and inequality (35). The corrected equation and inequality are shown as follows:

$$
\begin{aligned}
\frac{d V}{d t} & \\
= & n_{h}\left(1-n_{h}\right)\left(2-\frac{n_{h *}}{n_{h}}-\frac{s_{h *}}{s_{h}}\right) \\
& +\alpha s_{h *}\left(2-\frac{n_{h}}{n_{h *}}-\frac{s_{h}}{s_{h *}}\right) \\
& +\beta s_{h *} i_{v *}\left(3-\frac{i_{h}}{i_{h *}}-\frac{n_{h} i_{v *}}{n_{h *} i_{v}}-\frac{s_{h} i_{h *} i_{v}}{s_{h *} i_{h} i_{v *}}\right) \\
& +\beta s_{h *} i_{v *}\left(1-\frac{i_{v *}}{i_{v}}\right)\left(\frac{i_{v}}{i_{v *}}-\frac{n_{h}}{n_{h *}}\right) \\
& +\rho i_{h *}\left(\frac{n_{h *}}{n_{h}}-1\right)\left(\frac{n_{h}}{n_{h *}}+\frac{i_{h}}{i_{h *}}\right) \\
& +n_{v *} i_{v *}\left(\frac{n_{v} i_{h}}{n_{v *} i_{h *}}-\frac{i_{v}}{i_{v *}}\right)\left(1-\frac{i_{v *}}{i_{v}}\right) \\
& -\frac{1}{\varepsilon}\left(n_{v}-n_{v *}\right)^{2}-i_{h *} i_{v *}\left(1-\frac{i_{h}}{i_{h *}}\right)\left(1-\frac{i_{v}}{i_{v *}}\right) . \\
(2- & \left.\frac{n_{h *}}{n_{h}}-\frac{s_{h *}}{s_{h}}\right) \leq 0, \\
(2- & \left.\frac{n_{h}}{n_{h *}}-\frac{s_{h}}{s_{h *}}\right) \leq 0, \\
(3- & \left.\frac{i_{h}}{i_{h *}}-\frac{n_{h} i_{v *}}{n_{h *} i_{v}}-\frac{s_{h} i_{h *} i_{v}}{s_{h *} i_{h} i_{v *}}\right) \leq 0 .
\end{aligned}
$$

\section{References}

[1] A. A. Gebremeskel, "Global stability of malaria transmission dynamics model with logistic growth," Discrete Dynamics in Nature and Society, vol. 2018, Article ID 5759834, 12 pages, 2018. 


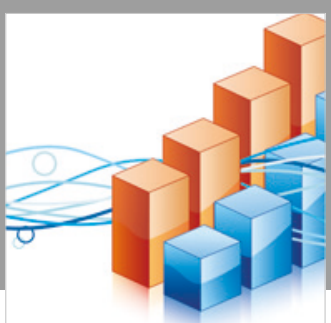

Advances in

Operations Research

\section{-n-m}
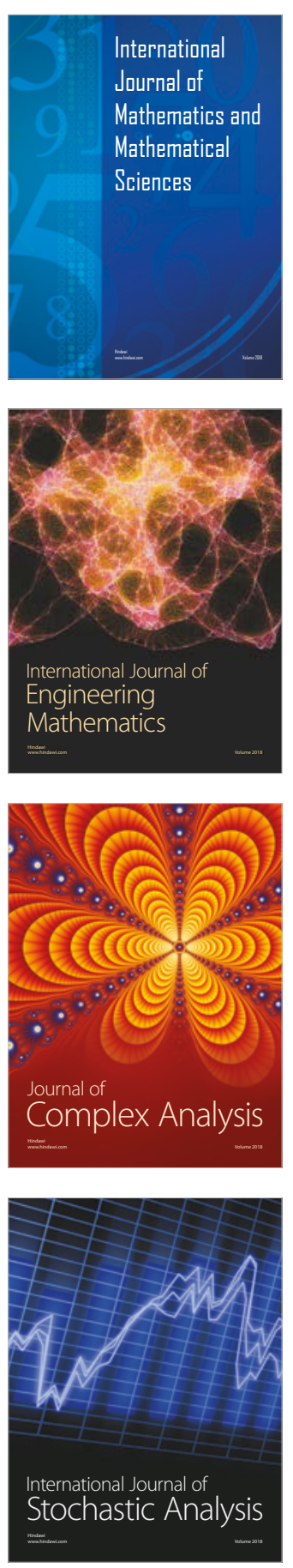
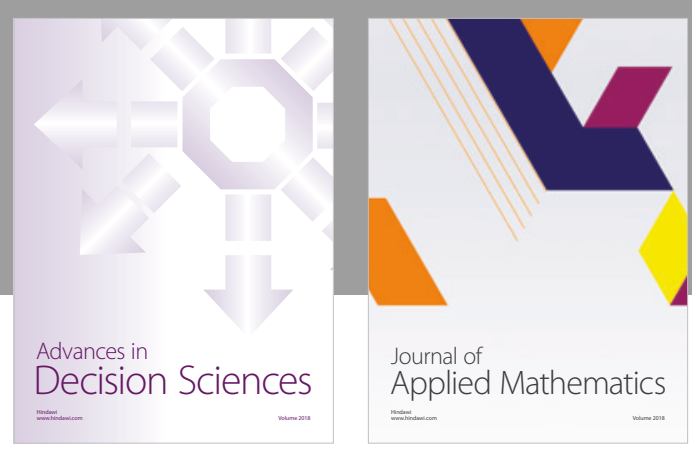

Journal of

Applied Mathematics
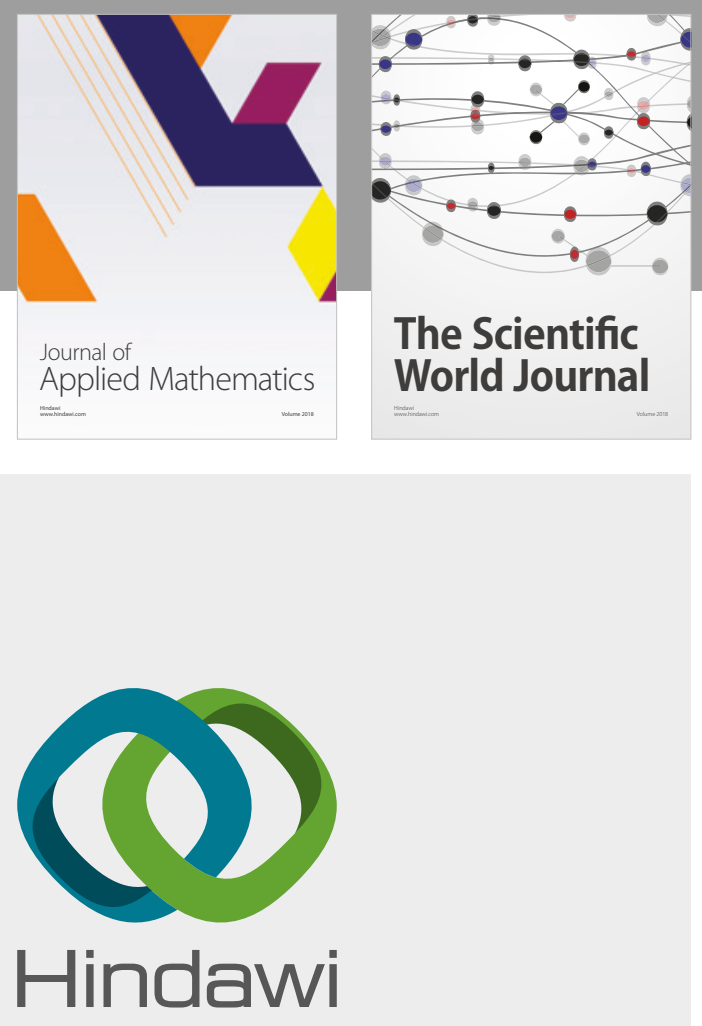

Submit your manuscripts at

www.hindawi.com

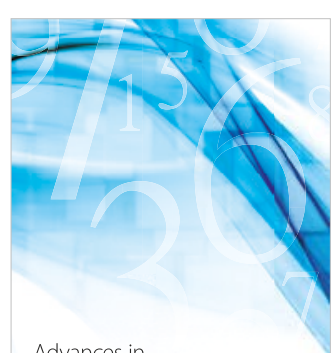

Advances in
Numerical Analysis
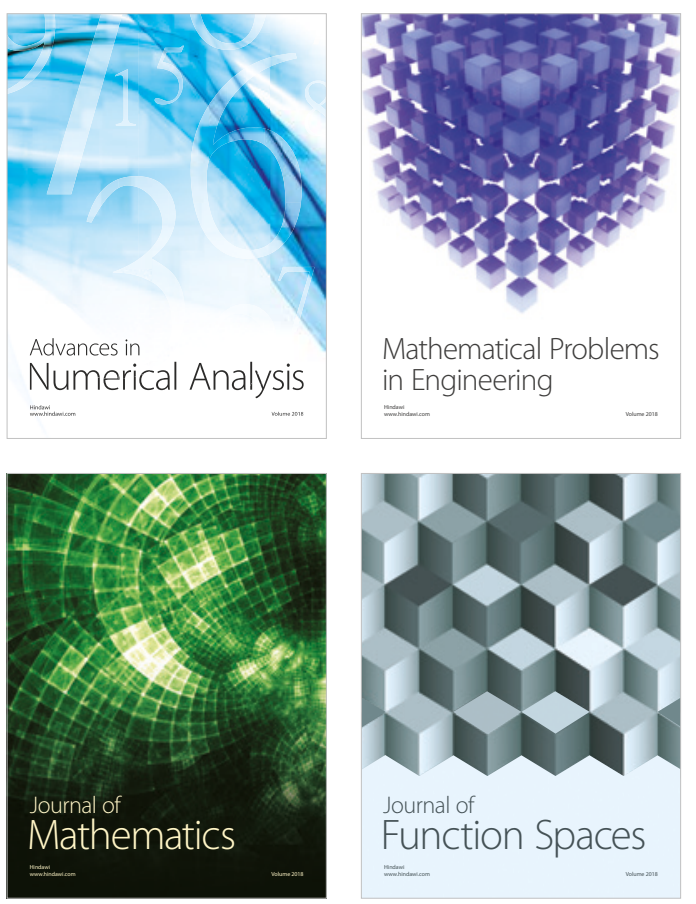

Mathematical Problems in Engineering

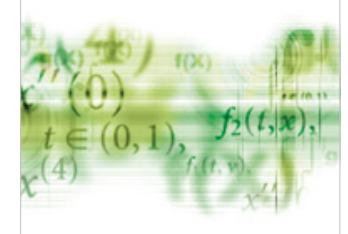

International Journal of

Differential Equations

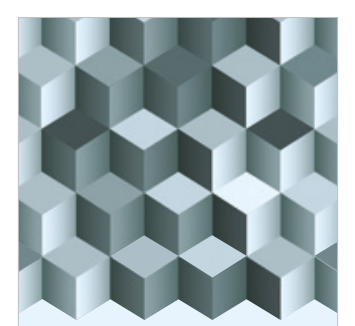

Journal of

Function Spaces
The Scientific

World Journal

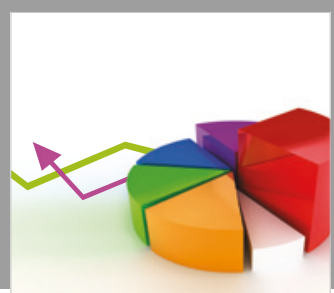

Journal of

Probability and Statistics
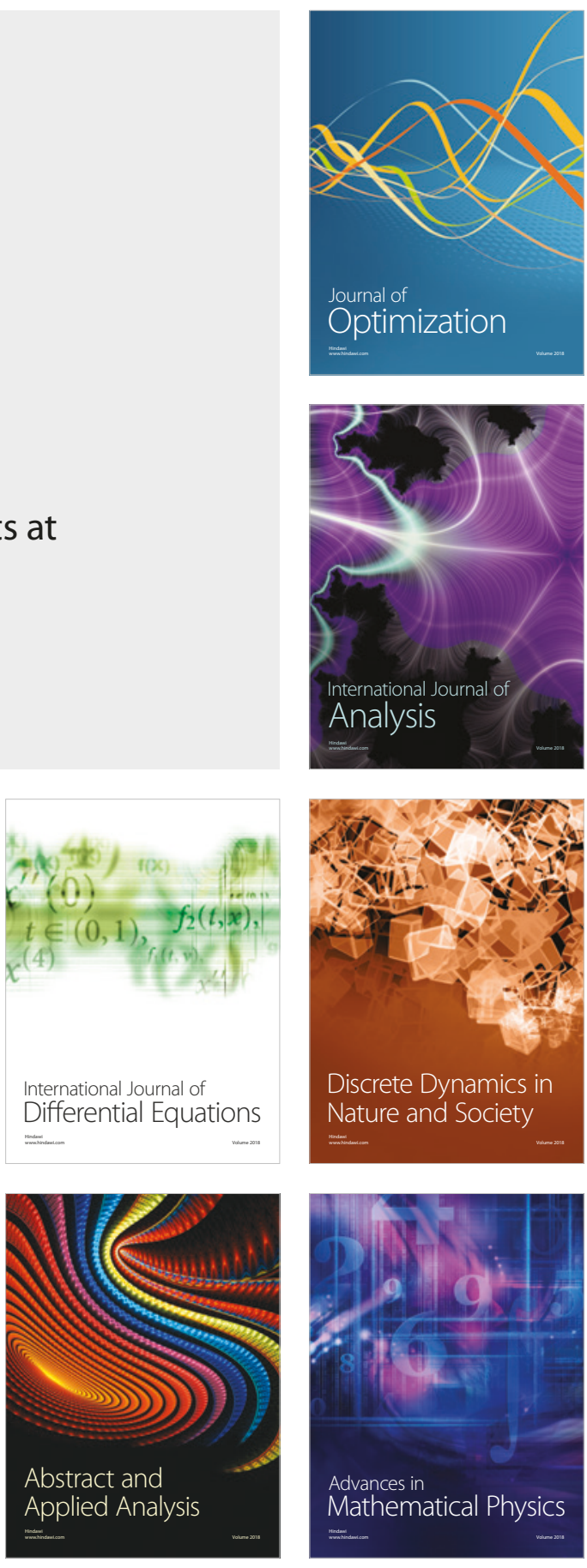